Pacific Journal of Mathematic 


\section{CHARACTERISTIC IDEALS IN GROUP ALGEBRAS}

\section{SinHA}

If $\mathfrak{F} G$ is the group-algebra of a group $G$ over a field $\mathfrak{F}$, and $\mathfrak{A}$ is any subgroup of the automorphism group of the $\mathfrak{F}$-algebra $\mathfrak{F} G$, then an ideal $I$ of $\mathfrak{F} G$, is called $\mathfrak{H}$-characteristic if $I^{\alpha} \leqq I, \quad \forall^{\alpha} \in A$. If $A$ is the whole automorphism group itself, then we merely say that $I$ is characteristic. Then D.S. Passman has proved the following result:

"Let $H \unlhd G$ such that $G / H$ is $\mathfrak{F}$-complete. Then for each characteristic ideal $I$ of $\mathfrak{F} G, I=(I \cap \widetilde{\mho} H) \mathfrak{F} G$."

The main concern in this paper is to consider the converse of this result.

2. Some preliminaries. For a given ideal $I \unlhd \mathfrak{F} G$, let $\mathscr{R}(I)$ be the set of all $H \leqq G$ such that $I=(I \cap \mathfrak{F} H) \mathfrak{F} G$. Let $C(I)$ be the set of all $H$ in $G$ such that if for some right $\mathfrak{F} H$-module $\mathfrak{M}, I \cap \mathfrak{\mho} H \cong$ Ann $\mathfrak{M}$, then $I \subseteq$ Ann $\mathfrak{M}^{G}$, the induced $\mathfrak{F} G$-module. We first of all have:

THEOREM 1. (i) For any $I \unlhd \mathfrak{F} G, C(I) \subseteq \mathscr{R}(I)$.

(ii) If $H \unlhd G$, then $H \in \mathscr{R}(I)$ if and only if $H \in C(I)$.

Proof. (i) Let $I \cap \mathfrak{F} H \subseteq$ Ann $\mathfrak{M}$ imply that $I \subseteq$ Ann $\mathfrak{M}^{G}$. Let $\sum p_{i} x_{i} \in I$ with $p_{\imath} \in \mathfrak{F} H$, where $G=\cup H x_{i}$ is a coset-decomposition. We have $\left(\sum \mathfrak{M} \otimes x_{i}\right)\left(\sum p_{i} x_{i}\right)=0$ if $I \cap \mathfrak{F} H \subseteq$ Ann $\mathfrak{M}$. In particular $(m \otimes I)\left(\sum p_{i} x_{i}\right)=0, \forall m \in \mathfrak{M}$, i.e., $\sum m p_{i} \otimes x_{i}=0, \forall m \in \mathfrak{M}$. So $\mathfrak{M} \cdot p_{i}=0$ for each $i$. Thus $p_{\imath} \in A$ nn $\mathfrak{M}$. Since $\mathfrak{M}$ is arbitrary with the property that $I \cap \mathfrak{F} H \subseteq$ Ann $\mathfrak{M}$, so we may take $\mathfrak{M}=\mathfrak{F} H / I \cap \mathfrak{F} H$, and conclude that each $p_{i} \in$ Ann $\mathfrak{M}=I \cap \mathfrak{F} H$. Thus $\sum p_{i} x_{i} \in(I \cap \mathfrak{F} H) \mathfrak{F} G$.

(ii) Suppose $I=\mathfrak{F} G(I \cap \mathfrak{F} H)$ and $I \cap \mathfrak{F} H \subseteq$ Ann $\mathfrak{M}$, for some $\mathfrak{F} H$ module $\mathfrak{M}$. Note that $H \unlhd G$ implies that $\mathfrak{F} G(I \cap \mathfrak{F} H)=(I \cap \mathfrak{F} H) \mathfrak{F} G$. Let $a=\sum x_{i} p_{\imath} \in I$ where $p_{i} \in I \cap \mathfrak{\mho} H . \quad$ So $a \mathfrak{M}^{\sigma}=\left(\sum x_{i} p_{i}\right)\left(\sum x_{j} \otimes \mathfrak{M}\right)=$ $\sum x_{i} x_{j} \otimes p_{i}^{x} j \mathfrak{M}=0$ since $p_{i}^{x} j \in I \cap \mathfrak{F} H \subseteq$ Ann $\mathfrak{M}$. Thus $a \mathfrak{M}^{G}=0$ and $I \subseteq$ Ann $\mathfrak{M}^{a}$.

Theorem 17.4 of [1] then gives us:

Corollary 1. Let $H \unlhd G$ such that $G / H$ is $\preccurlyeq$-complete. Then $H \in C(I)$ for every characteristic ideal $I$ of $\mathfrak{\mho} G$.

Also Theorem 17. 7 of [1] implies:

CoRollary 2. If $H \unlhd G \ni G / H$ is abelian and has no elements of order $p=$ Char. F, then $H \in C(J(G))$, where $J$ denotes the 


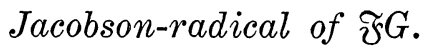

3. Main result. We will prove:

THEOREM 2. For $I=[\mathfrak{F} G, \mathfrak{F} G]$, the commutator ideal and for $J=J(G)$, if $H \leqq G$ such that $H \in \mathscr{R}(I)$ and $H \in \mathscr{R}(J)$ then $H \unlhd G$, $G / H$ is abelian with no elements of order $p$. In particular, $\mathfrak{F}(G / H)$ is semi-simple.

Further,if $\mathfrak{F}$ is algebraically closed then $G / H$ is $\mathfrak{F}$-complete.

We observe that the last two statements in the theorem follows from 17.8 and 17.1 (i) respectively of [1]. The rest of the theorem will be proved by a series of results proved below.

Lemma 1 . Let $H \leqq G, I \supseteqq \Re G$ and $H \in \mathscr{R}(I)$. Then $H \supseteqq \Re^{-1}(I)=$ $\{g \in G \mid g-1 \in I\}$.

Proof. Let $G=\cup H x_{i}$ be a coset-decomposition, and $g \in \mathfrak{P}^{-1}(I)$ such that $g \notin H$. Then $g=h x_{i}$ for some $i$, where $x_{i} \neq 1$, and $h \in H$;

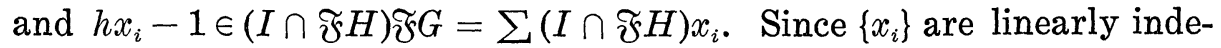
pendent over $\mathfrak{F} H, h \in I \cap \mathfrak{F} H$, and $x_{i} \neq 1$, so $g \in I$ which implies that $1 \in I$, a contradiction.

Lemma 2. If $I=[\mathfrak{\mho} G, \mathfrak{F} G]$, and $H \in \mathscr{R}(I)$ then $H \unlhd G$ and $G / H$ is abelian.

Proof. Observe that $I$ is a proper ideal in $\mathfrak{F} G$, since $\mathfrak{2}(I)=0$. Also by Lemma $1, H \supseteqq \mathfrak{A}^{-1}(I)$. Since $\left(g h g^{-1} h^{-1}-1\right) h g=g h-h g \in I$, for all $g, h \in G$, so $\left(g h g^{-1} h^{-1}-1\right) \in I$. Hence $g h g^{-1} h^{-1} \in \mathfrak{U}^{-1}(I) \subseteq H$; i.e., $G^{\prime}$, the commutator-subgroup is in $H$. Hence $H \unlhd G$ and $G / H$ is abelian.

Now let $H$ satisfy the hypothesis of Lemma 2. Then we have:

Lemma 3. Let $I=J(G)$ and $H \in \mathscr{R}(I)$. Then $\mathfrak{F}(G / H)$ is semisimple and $G / H$ has no elements of order $p=$ Char. $\mathfrak{F}$.

Proof. $J(G)=(J(G) \cap \mathfrak{F} H) \mathfrak{\Im} G \leqq J(H) \cdot \mathfrak{F} G$ by 16.9 of [1]. Now $\mathfrak{F} H\left[\mathfrak{X}_{H}(H) \cong \mathfrak{F}\right.$ where $\mathfrak{A}_{H}(H)$ is the ideal of $\mathfrak{F} H$, generated by $\{h-1 \mid h \in H\}$. So $\mathfrak{A}_{H}(H) \supseteqq J(H)$. Hence $\mathfrak{A}_{H}(H) \mathfrak{\Im} G=\mathfrak{A}_{G}(H) \supseteqq$ $J(H) \cdot \mathfrak{F} G \supseteqq J(G)$, where $\mathfrak{A}_{G}(H)$ is the ideal in $\mathfrak{F} G$, generated by $\{h-1 \mid h \in H\}$. Now $\mathfrak{N}_{G}(H)$ is the kernel of the natural map of $\mathfrak{F} G$ onto $\mathfrak{F}(G / H)$; \{see for example proof of Theorem 1 in [2]\}. Thus $\mathfrak{\mho}(G / H) \cong \widetilde{\Im} G / \mathfrak{2}_{G}(H)$ is semi-simple. Since $G / H$ is abelian by Lemma 
2, so it is clear that it has no elements of order $p$, as $\mathfrak{F}(G / H)$ is semi-simple.

This also completes the proof of Theorem 2 .

\section{REFERENCES}

1. D. S. Passman, Infinite Group Rings, Marcel Dekkar Inc., N.Y., 1971.

2. I. Sinha, On the augmentation-maps of subgroups of a group, Math. Zeitschs., 94 (1966), 193-206.

Received April 18, 1973.

Michigan State University 



\section{PACIFIC JOURNAL OF MATHEMATICS}

EDITORS

RICHARD ARENS (Managing Editor)

University of California

Los Angeles, Calıfornia 90024

R. A. Beaumont

University of Washington

Seattle, Washington 98105
J. DugundJI

Department of Mathematics

University of Southern California

Los Angeles, California 90007

D. Gilbarg and J. Milgram

Stanford University

Stanford, California 94305

\section{ASSOCIATE EDITORS}
E. F. BECKENBACH
B. H. NeumanN
F. WOLF
K. YOSHIDA

\section{SUPPORTING INSTITUTIONS}

UNIVERSITY OF BRITISH COLUMBIA

CALIFORNIA INSTITUTE OF TECHNOLOGY

UNIVERSITY OF CALIFORNIA

MONTANA STATE UNIVERSITY

UNIVERSITY OF NEVADA

NEW MEXICO STATE UNIVERSITY

OREGON STATE UNIVERSITY

UNIVERSITY OF OREGON

OSAKA UNIVERSITY
UNIVERSITY OF SOUTHERN CALIFORNIA

STANFORD UNIVERSITY

UNIVERSITY OF TOKYO

UNIVERSITY OF UTAH

WASHINGTON STATE UNIVERSITY

UNIVERSITY OF WASHINGTON

AMERICAN MATHEMATICAL SOCIETY NAVAL WEAPONS CENTER 


\section{Pacific Journal of Mathematics}

\section{Vol. 55, No. $1 \quad$ September, 1974}

Robert Lee Anderson, Continuous spectra of a singular symmetric

differential operator on a Hilbert space of vector-valued functions . . . $\quad 1$

Michael James Cambern, The isometries of $L^{p}(X, K) \ldots \ldots \ldots \ldots \ldots . . \ldots$

R. H. Cameron and David Arne Storvick, Two related integrals over spaces of continuous functions ................................

Gary Theodore Chartrand and Albert David Polimeni, Ramsey theory and

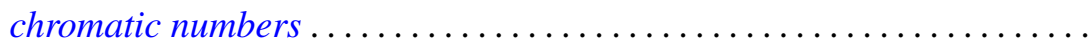

John Deryck De Pree and Harry Scott Klein, Characterization of collectively compact sets of linear operators ...................

John Deryck De Pree and Harry Scott Klein, Semi-groups and collectively compact sets of linear operators ....................... 55

George Epstein and Alfred Horn, Chain based lattices.............. 65

Paul Erdős and Ernst Gabor Straus, On the irrationality of certain series . . 85

Zdeněk Frolík, Measurable uniform spaces................... 93

Stephen Michael Gagola, Jr., Characters fully ramified over a normal

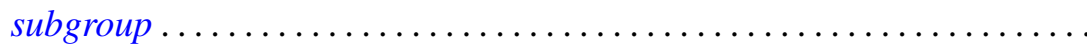

Frank Larkin Gilfeather, Operator valued roots of abelian analytic

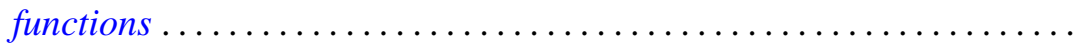

D. S. Goel, A. S. B. Holland, Cyril Nasim and B. N. Sahney, Best approximation by a saturation class of polynomial operators

James Secord Howland, Puiseux series for resonances at an embedded

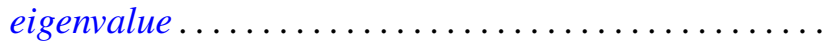

David Jacobson, Linear GCD equations .................

P. H. Karvellas, A note on compact semirings which are multiplicative

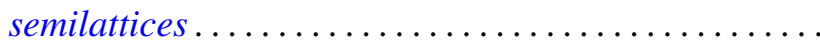

Allan Morton Krall, Stieltjes differential-boundary operators. II . .

D. G. Larman, On the inner aperture and intersections of convex sets

S. N. Mukhopadhyay, On the regularity of the $P^{n}$-integral and its application to summable trigonometric series ....... .

Dwight Webster Read, On $(J, M, m)$-extensions of Boolean algebras ....

David Francis Rearick, Multiplicativity-preserving arithmetic power series.

Indranand Sinha, Characteristic ideals in group algebras

Charles Thomas Tucker, II, Homomorphisms of Riesz spaces . . .

Kunio Yamagata, The exchange property and direct sums of indecomposable injective modules. 